\section{Diabetic mastopathy: imaging features and the role of image-guided biopsy in its diagnosis}

Jonghyeon Kim, Eun-Kyung Kim, Min Jung Kim, Hee Jung Moon, Jung Hyun Yoon

Department of Radiology, Research Institute of Radiological Science, Yonsei University

College of Medicine, Seoul, Korea

Purpose: The goal of this study was to evaluate the imaging features of diabetic mastopathy (DMP) and the role of image-guided biopsy in its diagnosis.

Methods: Two experienced radiologists retrospectively reviewed the mammographic and sonographic images of 19 pathologically confirmed DMP patients. The techniques and results of the biopsies performed in each patient were also reviewed.

Results: Mammograms showed negative findings in 78\% of the patients. On ultrasonography (US), 13 lesions were seen as masses and six as non-mass lesions. The US features of the mass lesions were as follows: irregular shape (69\%), oval shape (31\%), indistinct margin (69\%), angular margin (15\%), microlobulated margin (8\%), well-defined margin (8\%), heterogeneous echogenicity $(62 \%)$, hypoechoic echogenicity $(38 \%)$, posterior shadowing $(92 \%)$, parallel orientation (100\%), the absence of calcifications (100\%), and the absence of vascularity (100\%). Based on the US findings, 17 lesions (89\%) were classified as Breast Imaging Reporting and Data System category 4 and two (11\%) as category 3. US-guided core biopsy was performed in 18 patients, and 10 (56\%) were diagnosed with DMP on that basis. An additional vacuumassisted biopsy was performed in seven patients and all were diagnosed with DMP.

Conclusion: The US features of DMP were generally suspicious for malignancy, whereas the mammographic findings were often negative or showed only focal asymmetry. Core biopsy is an adequate method for initial pathological diagnosis. However, since it yields non-diagnostic results in a considerable number of cases, the evaluation of correlations between imaging and pathology plays an important role in the diagnostic process.

Keywords: Breast; Diabetes mellitus; Image-guided biopsy; Ultrasonography, mammography

\section{Introduction}

Diabetic mastopathy (DMP) is an uncommon benign fibrotic disease of the breast in patients with a history of long-standing diabetic mellitus (DM). It was first described as a breast lump consisting of fibrous tissue and lymphocytic infiltration in 12 patients with type I DM [1]. Since its first description, DMP has been widely recognized as a complication of type I DM. However, some recent reports have demonstrated that DMP can also be associated with type II DM $[2,3]$. One of the earliest studies of

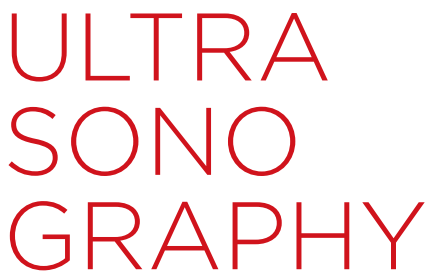

ORIGINAL ARTICLE

http://dx.doi.org/10.14366/usg. 15052 pISSN: 2288-5919 • elSSN: 2288-5943

Ultrasonography 2016;35:140-147

Received: September 3, 2015

Revised: November 9, 2015

Accepted: November 10, 2015

Correspondence to:

Eun-Kyung Kim, MD, Department of Radiology, Research Institute of Radiological Science, Yonsei University College of Medicine, 50-1 Yonsei-ro, Seodaemun-gu, Seoul 03722, Korea

Tel. +82-2-2228-2371

Fax. +82-2-393-3035

E-mail: ekkim@yuhs.ac

This is an Open Access article distributed under the terms of the Creative Commons Attribution NonCommercial License (http://creativecommons.org/ licenses/by-nc/3.0/) which permits unrestricted noncommercial use, distribution, and reproduction in any medium, provided the original work is properly cited.

Copyright () 2016 Korean Society of Ultrasound in Medicine (KSUM)

How to cite this article:

Kim J, Kim EK, Kim MJ, Moon HJ, Yoon JH. Diabetic mastopathy: imaging features and the role of image-guided biopsy in its diagnosis. Ultrasonography. 2016 Apr;35(2):140-147. 
DMP reported the imaging findings of DMP in 36 patients with longstanding type I DM and cataloged some helpful imaging findings, such as radiodense glandular tissue, strong acoustic shadowing in ultrasonography (US), and firm resistance to needle motion during the fine-needle aspiration (FNA) procedure [4]. Subsequent studies have reported similar findings, including heterogeneously dense breast parenchyma, dense glandular tissue or asymmetry in mammograms, and irregular hypoechoic solid lesions with posterior acoustic shadowing in US. However, those studies had limited generalizability due to the small number of cases available $[2,5]$.

DMP often presents as a palpable mass, but it may also be asymptomatic and is sometimes found incidentally during screening mammography or US. The imaging features are often suspicious for malignancy, meaning that biopsy is necessary. FNA is the least invasive option for the diagnosis of breast lesions, but it frequently provides inadequate specimens, which is why core needle biopsy (CNB) is the preferred method for diagnosing potentially malignant breast lesions $[4,6,7]$. To the best of our knowledge, no research has analyzed the imaging findings of DMP based on the Breast Imaging Reporting and Data System (BI-RADS), and to our knowledge, no studies have evaluated the role of image-guided biopsy in the diagnosis of DMP.

Therefore, the purpose of this study was to evaluate the imaging features of DMP and the role of image-guided biopsy in its diagnosis.

\section{Materials and Methods}

This retrospective review of images and medical records was approved by the Institutional Review Board of our institution, and the requirement for informed patient consent was waived. The pathology database of our institution-a tertiary medical center-was searched for cases with the keyword 'diabetic mastopathy' between 2004 and 2014. A total of 19 patients who underwent both imaging studies and biopsy at our institution were ultimately included in our study.

\section{Mammography and US}

Bilateral mammograms were obtained using full-field digital mammography systems (Selenia, Lorad/Hologic, Danbury, CT, USA; Senographe 2000D, GE Medical Systems, Milwaukee, WI, USA), except for one patient who underwent mammography before 2005 using another system (DMR, GE Medical Systems). Standard craniocaudal and mediolateral oblique views were routinely obtained. Additional mammographic views were obtained as needed. Bilateral whole breast US was performed by one of 18 radiologists with one to 17 years of experience in breast imaging with knowledge of each patient's clinical history and mammographic findings. High-resolution US was performed with either 7.5- or 12$\mathrm{MHz}$ linear array transducers, selected based on their availability (HDI 5,000 or iU, Philips-Advanced Technology Laboratories, Bothell, WA USA; Logic 9, GE Medical Systems).

\section{Image-Guided Biopsy}

US-CNB was performed with a 14-gauge automated core needle with a 22-mm throw (Stericut with coaxial, TSK Laboratory, Tochigi, Japan). At least five core samples were obtained from each lesion. US-guided vacuum-assisted biopsy (US-VAB) was performed using an 8- or 11-gauge needle (Mammotome, Ethicon Endo-Surgery, Cincinnati, $\mathrm{OH}, \mathrm{USA}$ ).

\section{Analysis of Images and Medical Records}

The mammograms and sonograms were retrospectively reviewed by two experienced radiologists (J.H.Y. and E.-K.K.) with seven and 18 years of experience in breast imaging, respectively, who arrived at a consensus for each image based on the fifth edition of BI-RADS. Only one pathologically confirmed lesion from each patient was included in this study. Lesions that did not appear as a mass on US were defined as non-mass lesions, although this terminology is not used in the fifth edition of BI-RADS. Medical records were reviewed to obtain clinical information. The clinical features recorded for each patient were the presence of symptoms, the type and duration of DM, DM medications, DM complications, and other medical history. Items not described in the medical records were marked as unknown and excluded from the analysis. Follow-up images and medical records were also reviewed to check for any changes of the DMP lesions over time.

\section{Results}

\section{Clinical Features}

All of the 19 patients were women, ranging in age from 28 to 74 years (mean age, 55 years). The clinical features of these patients are summarized in Table 1. Ten patients (53\%) presented with palpable masses, while the other nine were asymptomatic. Eleven patients (79\%) had type II DM, three had type I DM, and five had DM of unknown type. The mean duration of DM was 19.5 years (range, 4 to 39 years). Eleven patients had DM complications such as nephropathy and retinopathy. Other relevant conditions present in patients' medical histories included hypertension $(n=11)$ and hypothyroidism $(n=3)$.

\section{Mammographic and US Features}

Mammography was performed in 18 of the 19 patients, with the 
exception of a 28-year-old patient. All patients presented with heterogeneously dense $(n=14)$ or extremely dense $(n=4)$ breasts. Only four patients had abnormal findings on mammography, and all of the abnormalities involved asymmetry. None of the lesions manifested as distortions or suspicious microcalcifications on mammography.

The US findings of DMP are summarized in Table 2. The mean size of the lesions measured on US was $22.5 \mathrm{~mm}$ (range, 10 to 46 mm). On US, 13 cases had mass lesions (Fig. 1) while six cases had non-mass lesions (Fig. 2). All 13 mass lesions were solid, and their common US features were irregular shape $(n=9,69 \%)$, indistinct margin $(n=9,69 \%)$, heterogeneous echogenicity $(n=8,62 \%)$, posterior acoustic shadowing $(n=12,92 \%)$, parallel orientation $(n=13,100 \%)$, the absence of calcifications $(n=13,100 \%)$, and the absence of vascularity $(n=13,100 \%)$. None of the six non-mass lesions had calcifications, but all had posterior shadowing, with two

Table 1. The clinical features of 19 patients with diabetic mastopathy

\begin{tabular}{lc}
\multicolumn{1}{c}{ Feature } & No. $(\%)^{\text {a }}$ \\
\hline Symptom & $10(53)$ \\
Palpable mass & $9(47)$ \\
Asymptomatic & \\
DM type & $3(21)$ \\
Type I & $11(79)$ \\
Type II & 5 \\
Unknown & \\
DM complications & $2(15)$ \\
None & $4(31)$ \\
Nephropathy only & $4(31)$ \\
Retinopathy only & $3(23)$ \\
Nephropathy and retinopathy & 6 \\
Unknown & \\
DM medications & $6(40)$ \\
Insulin only & $2(13)$ \\
Insulin and oral medication & $6(40)$ \\
Oral medication only & $1(7)$ \\
None & 4 \\
Unknown & $11(61)$ \\
Other medical history & $3(17)$ \\
Hypertension & $4(22)$ \\
None & 1 \\
Unknownyroidism & \\
\hline
\end{tabular}

a) Unknown cases were excluded from the calculation of percentage. DM, diabetes mellitus. being intense. None of the patients had enlarged axillary lymph nodes. Multiple similar lesions were seen in nine patients, and seven patients had lesions in both breasts. Based on the US findings, 11 of the 13 mass lesions and all six of the non-mass lesions were classified as BI-RADS category 4 , with 13 cases classified as $4 A$, three as $4 B$, and one as $4 C$. The remaining two were classified as $\mathrm{BI}$ RADS category 3 (Table 3 ).

\section{Pathologic Diagnosis and Follow-up}

All 19 patients were pathologically diagnosed with DMP. Ten were diagnosed via US-CNB, eight via US-VAB, and one through surgical excision (Table 3). Among the eight patients who were diagnosed with DMP using US-VAB, seven underwent US-CNB before US$V A B$. Initial US-CNB found nonspecific fibrosis in four of these seven

Table 2. Ultrasonographic features of the 13 patients with diabetic mastopathy presenting as a mass lesion

\begin{tabular}{|c|c|}
\hline Feature & No. (\%) \\
\hline \multicolumn{2}{|l|}{ Shape } \\
\hline Oval & $4(31)$ \\
\hline Irregular & $9(69)$ \\
\hline \multicolumn{2}{|l|}{ Margin } \\
\hline Angular & $2(15)$ \\
\hline Indistinct & $9(69)$ \\
\hline Microlobulated & $1(8)$ \\
\hline Spiculated & 0 \\
\hline Well-defined & $1(8)$ \\
\hline \multicolumn{2}{|l|}{ Echogenicity } \\
\hline Heterogeneous & $8(62)$ \\
\hline Hypoechoic & $5(38)$ \\
\hline \multicolumn{2}{|l|}{ Posterior shadowing } \\
\hline Shadowing & $7(54)$ \\
\hline Intense shadowing & $5(38)$ \\
\hline No shadowing & $1(8)$ \\
\hline \multicolumn{2}{|l|}{ Vascularity } \\
\hline No & $13(100)$ \\
\hline \multicolumn{2}{|l|}{ Orientation } \\
\hline Parallel & $13(100)$ \\
\hline \multicolumn{2}{|l|}{ Calcifications } \\
\hline No & $13(100)$ \\
\hline \multicolumn{2}{|l|}{ BI-RADS category } \\
\hline 3 & $2(15)$ \\
\hline $4 \mathrm{~A}$ & $9(69)$ \\
\hline $4 B$ & $1(8)$ \\
\hline $4 \mathrm{~B}$ & $1(8)$ \\
\hline
\end{tabular}

BI-RADS, Breast Imaging Reporting and Data System. 


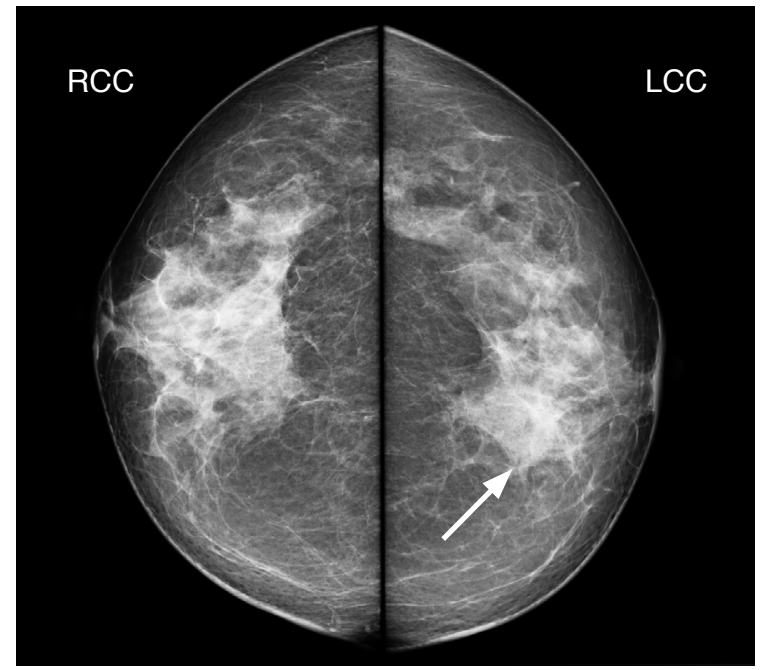

A

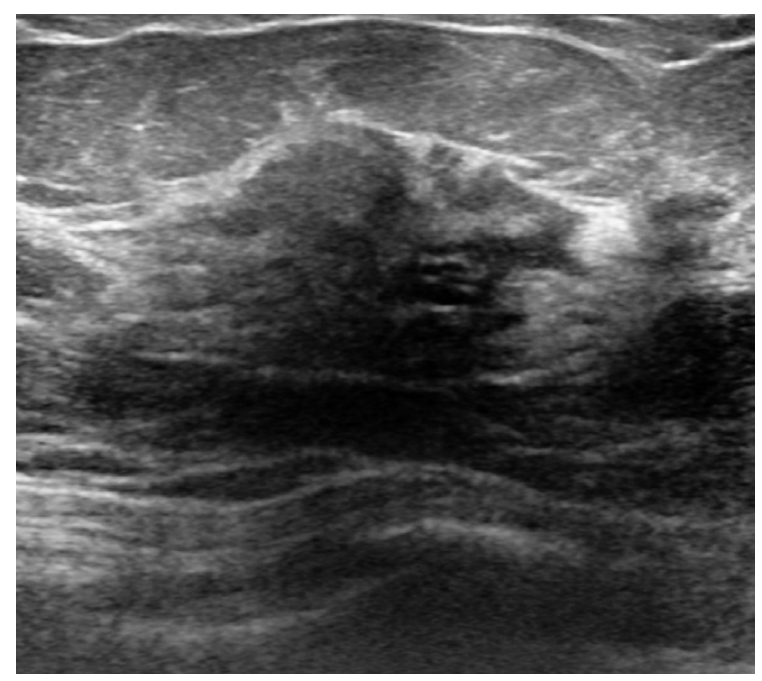

C

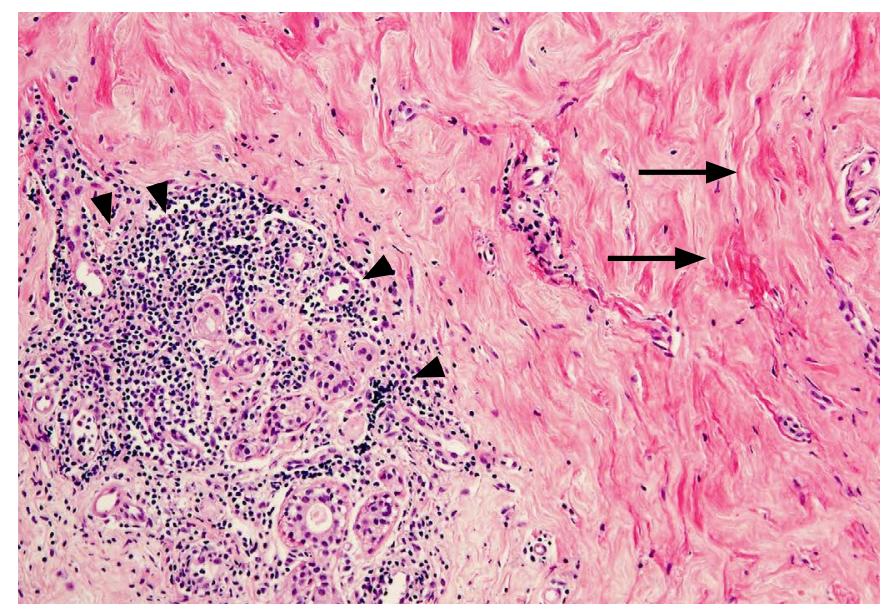

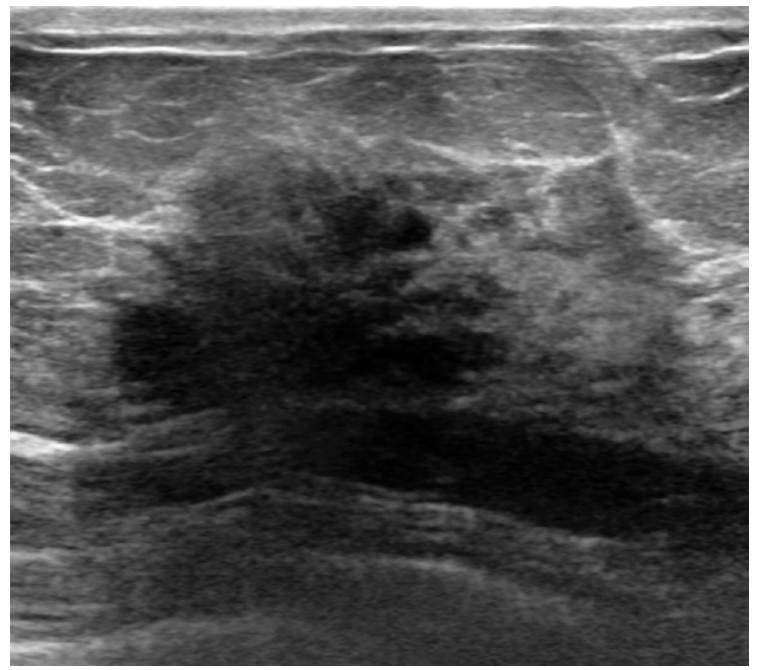

B

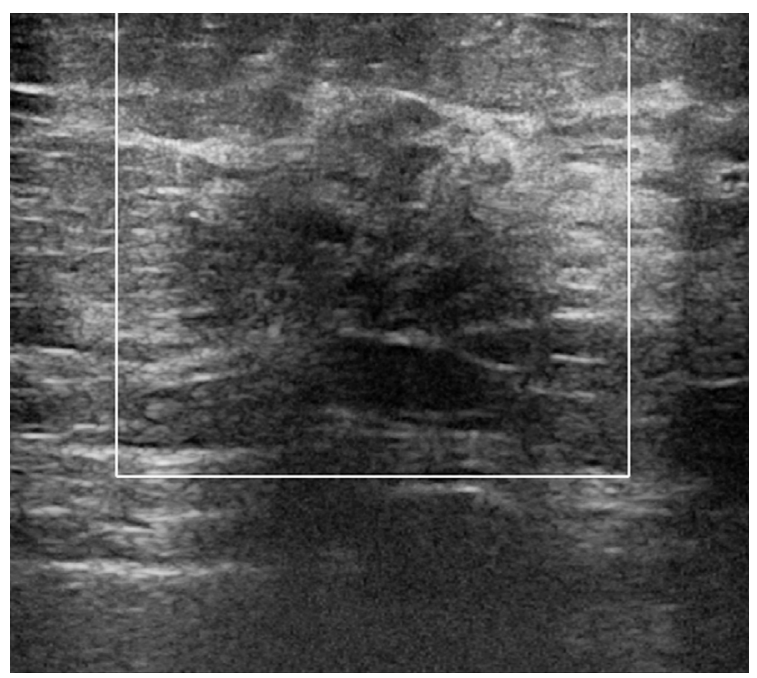

D

Fig. 1. A 64-year-old woman with type II diabetes mellitus complaining of a palpable lump in her left upper breast (case 12). A. Craniocaudal mammogram shows asymmetry in the left inner breast (arrow). LCC, left craniocaudal; RCC, right craniocaudal. $B, C$. Transverse (B) and longitudinal (C) sonograms demonstrate an approximately $2.1-\mathrm{cm}$ irregular hypoechoic mass with angular margins and posterior shadowing at the area of the palpable lump in the left upper inner breast. D. This lesion shows no vascularity. Ultrasonography-guided core needle biopsy was performed on this lesion, resulting in a diagnosis of diabetic mastopathy. $E$. The histopathologic specimen shows collagenous stroma with keloidal features (arrows) and periductal lymphocytic infiltration (arrowheads), favoring diabetic mastopathy $(H \& E, \times 100)$. 
patients, which was discordant with the imaging findings, leading to additional US-VAB. The US-CNB results of the remaining three cases were fibroadenoma, usual ductal hyperplasia, and marked sclerosis, and all were concordant with the imaging findings. Additional vacuum-assisted biopsy (VAB) was performed in one of these three cases because the lesion showed an increase in size at follow-up, and in the other two cases because the patient requested further diagnostic test results.

Twelve patients were followed up with US for a mean period of 44.4 months (range, 5.8 to 103.4 months) after the initial biopsy (Table 3). During the follow-up period, the lesion became larger in two patients, one of whom also developed additional lesions on both sides of the breast with similar characteristics to the original lesion. Therefore, core biopsies were performed once more on the lesion that had grown and on the new lesion, and the results confirmed DMP. The lesions disappeared in three of the seven patients who underwent $V A B$, shrunk in two patients, and remained the same size in two patients during the follow-up period. None of the patients were diagnosed with breast cancer during the followup period.

\section{Discussion}

The etiology and pathophysiology of DMP have not been adequately

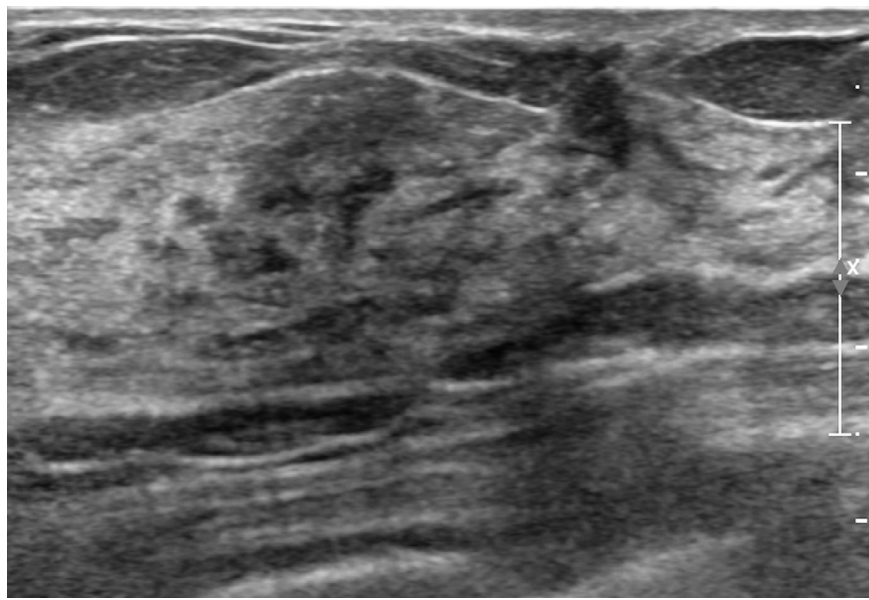

B

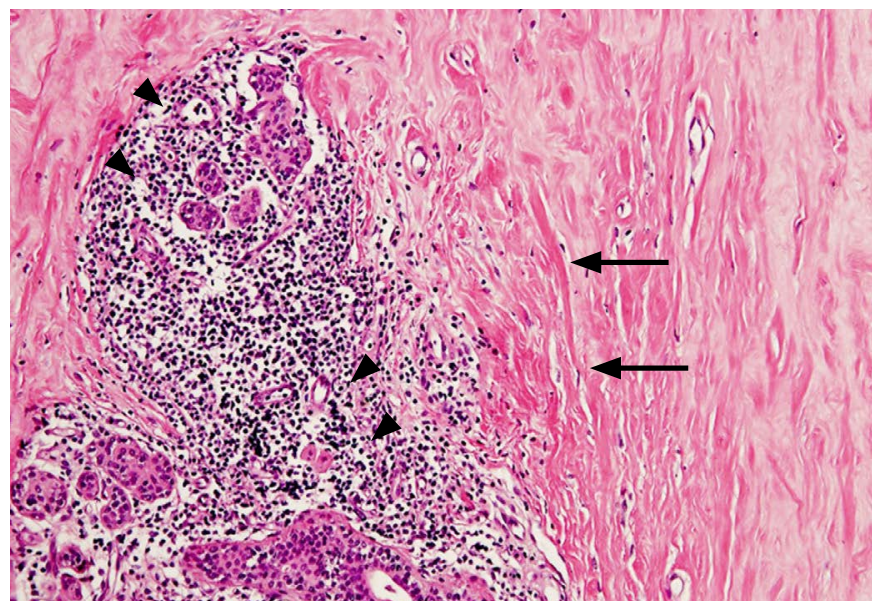

D

C

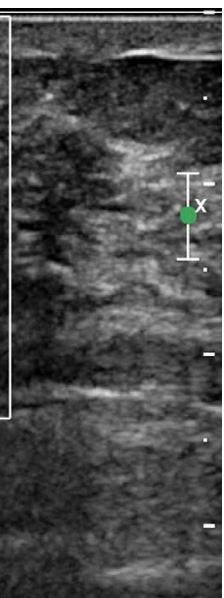

Fig. 2. A 57-year-old woman with type II diabetes mellitus complaining of a palpable lump in her right upper breast (case 10).

The mammogram showed heterogeneously dense breasts without remarkable findings in the area of the palpable lump (not shown). A, B. Transverse (A) and longitudinal (B) sonograms of the area with the palpable lump in the right breast show an ill-defined hypoechoic area with posterior shadowing that does not appear as a space-occupying mass. C. The lesion shows no vascularity. Ultrasonography-guided core needle biopsy was performed on this lesion. D. The histopathologic specimen shows keloid-like stromal fibrosis (arrows) and periductal lymphocytic infiltration (arrowheads) suggestive of diabetic mastopathy $\left(H \& E_{1} \times 100\right)$. 
Table 3. Pathologic diagnosis and follow-up results in 19 patients with diabetic mastopathy

\begin{tabular}{|c|c|c|c|c|c|c|c|c|c|c|}
\hline No. & Age (yr) & Palpability & Size $(\mathrm{mm})$ & $\begin{array}{l}\text { Lesion } \\
\text { type }\end{array}$ & $\begin{array}{l}\text { BI-RADS } \\
\text { category }\end{array}$ & CNB results & $\begin{array}{l}\text { Image-CNB } \\
\text { correlation }\end{array}$ & $\begin{array}{l}\text { VAB or surgery } \\
\text { results }\end{array}$ & $\begin{array}{l}\text { Follow-up } \\
\text { interval } \\
\text { (mo) }\end{array}$ & Follow-up results \\
\hline 1 & 68 & Yes & 23 & Mass & $4 \mathrm{~A}$ & DMP & Concordant & - & - & - \\
\hline 2 & 57 & No & 14 & Mass & $4 \mathrm{~A}$ & DMP & Concordant & - & - & - \\
\hline 3 & 66 & No & 19 & Mass & $4 \mathrm{~A}$ & DMP & Concordant & - & - & - \\
\hline 4 & 44 & Yes & 10 & Non-mass & $4 \mathrm{~A}$ & DMP & Concordant & - & - & - \\
\hline 5 & 49 & No & 19 & Non-mass & 3 & DMP & Concordant & - & - & - \\
\hline 6 & 51 & Yes & 21 & Mass & $4 \mathrm{~B}$ & $\begin{array}{l}\text { Usual ductal } \\
\text { hyperplasia }\end{array}$ & Concordant & DMP & - & - \\
\hline 7 & 50 & Yes & 24 & Non-mass & $4 \mathrm{~A}$ & $\begin{array}{l}\text { Nonspecific } \\
\text { fibrosis }\end{array}$ & Discordant & DMP (surgery) & - & - \\
\hline 8 & 28 & Yes & 20 & Mass & $4 \mathrm{~B}$ & DMP & Concordant & - & 61.3 & Decreased \\
\hline 9 & 33 & Yes & 46 & Non-mass & 3 & DMP & Concordant & - & 12.6 & Increased \\
\hline 10 & 57 & Yes & 27 & Non-mass & $4 \mathrm{~A}$ & DMP & Concordant & - & 12.4 & No change \\
\hline 11 & 74 & No & 24 & Non-mass & $4 \mathrm{~A}$ & DMP & Concordant & - & 5.8 & No change \\
\hline 12 & 64 & Yes & 21 & Mass & $4 \mathrm{~A}$ & DMP & Concordant & - & 19.9 & $\begin{array}{c}\text { No change } \\
\text { New lesions in both } \\
\text { breasts }\end{array}$ \\
\hline 13 & 43 & Yes & 24 & Mass & $4 \mathrm{~A}$ & - & - & DMP & 6.1 & No change \\
\hline 14 & 53 & Yes & 28 & Mass & $4 C$ & $\begin{array}{l}\text { Marked } \\
\text { sclerosis }\end{array}$ & Concordant & DMP & 103.4 & Decreased \\
\hline 15 & 59 & No & 19 & Mass & $4 \mathrm{~B}$ & $\begin{array}{l}\text { Nonspecific } \\
\text { fibrosis }\end{array}$ & Discordant & DMP & 65.6 & Disappeared \\
\hline 16 & 58 & No & 12 & Mass & $4 \mathrm{~A}$ & $\begin{array}{l}\text { Nonspecific } \\
\text { fibrosis }\end{array}$ & Discordant & DMP & 49.0 & $\begin{array}{c}\text { Disappeared } \\
\text { New lesion in the } \\
\text { contralateral breast }\end{array}$ \\
\hline 17 & 58 & No & 24 & Mass & $4 \mathrm{~A}$ & Fibroadenoma & Concordant & DMP & 78.4 & $\begin{array}{c}\text { No change } \\
\text { New lesions in both } \\
\text { breasts }\end{array}$ \\
\hline 18 & 73 & No & 27 & Mass & $4 \mathrm{~A}$ & $\begin{array}{l}\text { Nonspecific } \\
\text { fibrosis }\end{array}$ & Discordant & DMP & 63.0 & Disappeared \\
\hline 19 & 67 & No & 25 & Mass & $4 \mathrm{~A}$ & $\begin{array}{l}\text { Nonspecific } \\
\text { fibrosis }\end{array}$ & Discordant & DMP & 55.1 & $\begin{array}{l}\text { New lesion in the } \\
\text { ipsilateral breast }\end{array}$ \\
\hline
\end{tabular}

BI-RADS, Breast Imaging Reporting and Data System; CNB, core needle biopsy; VAB, vacuum-assisted biopsy; DMP, diabetic mastopathy.

explained, and only hypotheses have been proposed. According to one hypothesis, hyperglycemia is the key etiology of DMP. Glycosylation is accelerated in hyperglycemia, and excessive levels of glycosylated end products induce an autoimmune response, causing lymphocytic infiltration and fibrosis. According to this theory, the severity and duration of hyperglycemia is an important predictive risk factor for DMP [8-13]. Another theory explains DMP as breast involvement of systemic autoimmune disease, based on the similarity of histological features observed in DMP lesions and lymphoepithelial lesions from Hashimoto thyroiditis or Sjögren syndrome [14]. Others have proposed that exogenous insulin itself, its delivery mechanism, or contaminants therein trigger an immune reaction that consequently results in DMP $[9,15]$. In the past, when studies of DMP were far more limited, DMP was thought to be a breast disease that specifically occurred in type I DM patients using exogenous insulin [15]. However, DMP has also been found in type II DM patients. In fact, this study, although there were five patients with DM of unknown type, included more type II DM patients $(n=11)$ than type I DM patients $(n=3)$. This epidemiological trend may reflect the increased prevalence of long-standing type II DM, due to its increased incidence and the prolonged survival period of type II DM patients.

The mammographic findings of DMP in our study, which were mostly negative findings in dense breast parenchyma, were consistent with those reported in previous studies [5,9]. In contrast, the US findings of DMP, which are discussed further below, were 
usually suspicious for malignancy. The presence of conflicting results between mammography and US may be helpful in diagnosing DMP.

The US findings varied from case to case, but the most typical US findings observed for DMP were an irregularly shaped, heterogeneous echoic mass with posterior shadowing, which was also consistent with previous studies [5]. These US findings, which were observed in the majority of DMP lesions, corresponded to a diagnosis of BI-RADS category 4, meaning that the biopsy was necessary to exclude malignant tumors from the differential diagnosis.

The US findings in six of the 19 lesions did not resemble masses (i.e., were not space-occupying lesions in two different dimensions), and we therefore classified them as non-mass lesions. This term is not present in the most recent BI-RADS US lexicon, and no standardized method exists for interpreting this kind of lesion. In clinical practice, however, lesions sometimes cannot be classified as masses since they do not meet the definitive criteria of masses. Non-mass-like enhancement is a well-known category in the breast magnetic resonance imaging lexicon, but this terminology has not been commonly used in breast US. However, after the Japan Association of Breast and Thyroid Sonology termed these lesions non-mass image-forming lesions, the term 'non-mass' has started to appear in published studies $[16,17]$. According to a recently published report regarding the US and histopathological findings of non-mass lesions, five cases of DMP were found among 164 nonmass lesions [18]. The authors classified the US findings of non-mass lesions into four types: type I lesions are non-mass lesions showing multiple duct-like structures arranged in a parallel orientation, type II lesions involve a mottled area that does not present as a discrete mass, type III lesions are non-mass lesions associated with architectural distortion, and type IV lesions are non-mass lesions presenting with posterior acoustic shadowing. The five DMP lesions found in this study all showed a type IV pattern on US, and the positive predictive value of type IV non-mass lesions for malignancy has been reported to be similar to that of BI-RADS category 4B. All of the six non-mass-like DMP lesions found in our study were also associated with posterior shadowing, falling into the category of type IV lesions. If type IV non-mass lesions can be equated to BIRADS category $4 B$, the pathologic result of DMP from these lesions can be considered concordant with the imaging findings.

Since it is currently impossible to differentiate between DMP and breast cancer based on imaging studies, pathological examination is necessary in most cases. A sample from the breast lesion can be obtained using several methods, and the least invasive procedure is usually preferable. However, FNA, which is considered to be the least invasive procedure, is not commonly used because it often yields inadequate specimens for the diagnosis of DMP [12]. The results of our study showed that CNB yielded a conclusive diagnosis in approximately half of the cases, as ten of the 18 patients who underwent CNB were diagnosed with DMP. Nonspecific fibrosis was the most common result among the cases of DMP that were not correctly diagnosed by initial CNB. In these cases, as CNB acquires only a small part of the lesion, it may have missed the site of inflammation, which is an important diagnostic clue for pathologists. However, CNB is still a better method for the initial biopsy of suspicious breast lesions than FNA or VAB with regard to reliability, feasibility, and risk.

The role of US-VAB in the diagnosis of DMP has not yet been studied. In our study, eight patients who did not receive a specific diagnosis based on initial CNB findings were pathologically diagnosed with DMP via $V A B$, meaning that surgical excision was fortunately avoided. When the initial biopsy of a suspicious lesion fails to yield a definitive diagnosis, re-biopsy generally requires a larger amount of breast tissue. We suggest that US-VAB is a useful method in this situation, as it provides a larger specimen while still being less invasive than surgical excision. Surgical excision is not required in cases of DMP, as DMP is a universally benign type of lesion. Furthermore, previous studies have stated that surgical biopsy should not be considered because the recurrence rate of the disease has been reported to be as high as 60\% [12,19].

This study had some limitations. First of all, it was a retrospective study, meaning that selection bias was inevitable. Since the study population was obtained by searching our institution's pathology database, patients with DMP who did not undergo biopsy, presumably due to benign-appearing imaging findings, were missed. In addition, since the sonograms were reviewed retrospectively, unmeasurable interperformer differences may have been present. Many patients had limited clinical information regarding their DM status, since their DM was managed in other clinics. Therefore, the overall incidence of DMP and its relationship with various clinical conditions, such as DM complications, medication, or length of hyperglycemia must be evaluated in other future studies with a more specific cohort of DM patients.

In conclusion, while the majority of DMP lesions showed negative findings or nonspecific focal asymmetry on mammograms, the US features were commonly suspicious for malignancy, corresponding to BI-RADS category 4 and making a pathologic diagnosis necessary. US-CNB is the method of choice for initial biopsy due to its feasibility and reliability. As CNB gives nonspecific results in some cases, assessing the correlation between imaging and pathology plays an important diagnostic role, and an additional biopsy may be required. In the cases included in this study, US-VAB sufficed for a conclusive diagnosis, and surgical excision was therefore avoided. 
ORCID: Jonghyeon Kim: http://orcid.org/0000-0001-6155-198X; Eun-Kyung Kim: http://orcid.org/0000-0002-3368-5013; Min Jung Kim: http://orcid.org/0000-00034949-1237; Hee Jung Moon: http://orcid.org/0000-0002-5643-5885; Jung Hyun Yoon: http://orcid.org/0000-0002-2100-3513

\section{Conflict of Interest}

No potential conflict of interest relevant to this article was reported.

\section{Acknowledgments}

This study was supported in part by the Research Fund of the Korean Society of Ultrasound in Medicine.

\section{References}

1. Soler NG, Khardori R. Fibrous disease of the breast, thyroiditis, and cheiroarthropathy in type I diabetes mellitus. Lancet 1984;1:193195.

2. Dorokhova O, Fineberg S, Koenigsberg T, Wang Y. Diabetic mastopathy, a clinicopathological correlation of 34 cases. Pathol Int 2012;62:660-664.

3. Pereira MA, de Magalhaes AV, da Motta LD, Santos AM, Segura $M E$, Pereira $C F$, et al. Fibrous mastopathy: clinical, imaging, and histopathologic findings of 31 cases. J Obstet Gynaecol Res 2010;36:326-335.

4. Logan WW, Hoffman NY. Diabetic fibrous breast disease. Radiology 1989;172:667-670.

5. Ricart Selma V, Camps Herrero J, Martinez Rubio C, Cano Munoz R, Gonzalez Noguera PJ, Forment Navarro M, et al. Diabetic mastopathy: clinical presentation, imaging and histologic findings, and treatment. Radiologia 2011;53:349-354.

6. Yu YH, Wei W, Liu JL. Diagnostic value of fine-needle aspiration biopsy for breast mass: a systematic review and meta-analysis. BMC Cancer 2012;12:41.

7. Andrews-Tang $D$, Diamond $A B$, Rogers $L$, Butler D. Diabetic mastopathy: adjunctive use of ultrasound and utility of core biopsy in diagnosis. Breast J 2000;6:183-188.

8. Moschetta M, Telegrafo M, Triggiani V, Rella L, Cornacchia I, Serio $\mathrm{G}$, et al. Diabetic mastopathy: a diagnostic challenge in breast sonography. J Clin Ultrasound 2015;43:113-117.

9. Chan $\mathrm{CL}$, Ho RS, Shek TW, Kwong A. Diabetic mastopathy. Breast J 2013;19:533-538.

10. Kojima T, Kammori M, Hashimoto M, Ogawa T, Yasuda H, Takazawa $Y$, et al. Diabetic mastopathy in an advanced elderly woman with insulin-dependent type 2 diabetes mellitus. Breast Cancer 2003; 10:374-377.

11. Ely KA, Tse G, Simpson JF, Clarfeld R, Page DL. Diabetic mastopathy: a clinicopathologic review. Am J Clin Pathol 2000;113:541-545.

12. Camuto PM, Zetrenne E, Ponn T. Diabetic mastopathy: a report of 5 cases and a review of the literature. Arch Surg 2000;135:11901193.

13. Tomaszewski JE, Brooks JS, Hicks D, Livolsi VA. Diabetic mastopathy: a distinctive clinicopathologic entity. Hum Pathol 1992;23:780786.

14. Schwartz IS, Strauchen JA. Lymphocytic mastopathy: an autoimmune disease of the breast? Am J Clin Pathol 1990;93:725-730.

15. Seidman JD, Schnaper LA, Phillips LE. Mastopathy in insulinrequiring diabetes mellitus. Hum Pathol 1994;25:819-824.

16. Uematsu T. Non-mass-like lesions on breast ultrasonography: a systematic review. Breast Cancer 2012;19:295-301.

17. Japan Association of Breast and Thyroid Sonology. Guideline for breast ultrasound: management and diagnosis. Tokyo: Nankodo Co., 2004;35-37, 53-60.

18. Ko KH, Hsu HH, Yu JC, Peng YJ, Tung HJ, Chu CM, et al. Non-masslike breast lesions at ultrasonography: feature analysis and BI-RADS assessment. Eur J Radiol 2015;84:77-85.

19. Thanarajasingam U, Chen B, Tortorelli CL, Jakub JW, Ghosh K. Diabetic mastopathy as a radiographically occult palpable breast mass. Case Rep Med 2011;2011:162350. 